\title{
Reproducción de Joturus pichardi y Agonostomus monticola (Mugiliformes: Mugilidae) en ríos de la Sierra Nevada de Santa Marta, Colombia
}

\author{
Pedro Eslava Eljaiek ${ }^{1} \&$ Roy Díaz Vesga ${ }^{2}$ \\ 1. Programa de Ingeniería Pesquera, Universidad del Magdalena, Carrera 32 No. 22-08 Santa Marta, Magdalena, \\ Colombia; ingpesqueraunimagdalena@gmail.com \\ 2. Laboratorio de Investigaciones Pesqueras Tropicales, Universidad del Magdalena, Carrera 32 No. 22-08 Santa Marta, \\ Magdalena, Colombia; rdiazvesga@gmail.com
}

\author{
Recibido 17-I-2011. Corregido 03-IV-2011. Aceptado 02-V-2011.
}

\begin{abstract}
Reproduction of Joturus pichardi and Agonostomus monticola (Mugiliformes: Mugilidae) in rivers of the Sierra Nevada de Santa Marta, Colombia. The freshwater mugilids Joturus pichardi and Agonostomus monticola, have been documented on ecological and distribution aspects, mainly for Central American populations, nevertheless, little information is available on their reproductive aspects, specifically in Colombian freshwater environments. Reproductive biology of the mugilids J. pichardi and A. monticola from Sierra Nevada de Santa Marta (SNSM) rivers was studied between July 2005 and December 2006. A total of 14 specimens of $J$. pichardi and 320 of A. monticola were collected. The reproductive biology was analyzed by means of: sexual proportion, gonadosomatic index, and mean size at maturity, fecundity and oocyte diameter. Additionally, a bioassay was carried out to evaluate the effect of salinity on spermatic motility and its possible relationship with the species' spawning area. These mugilids share habitats with similar ecological characteristics, in which strong currents; clear water and stony areas stand out. Gonadal maturity indicators and indirect evidence are presented to support the relationship between reproductive maturity and higher rainfall levels in the area (September, October and November), as well as the catadromous migration of J. pichardi and A. monticola. This last species females outnumbered males with a sex ratio of 2.3:1. Females mean size at maturity was $172 \mathrm{~mm}$ of their total length (TL) and $108 \mathrm{~mm}$ TL for males. Fecundity (F) was $23925 \pm 4581$ eggs per gram of gonad, and was related to size by the equation $\mathrm{F}=395.1 \mathrm{TL}^{1.281}$; besides, the mean oocyte diameter was $362 \pm 40 \mu \mathrm{m}$. Considering the salinity effect on sperm motility in both species, results suggested that $J$. pichardi spawned in estuarine environments but the species did not migrate to fully marine environments; however, A. monticola withstood a broad range of salinity, suggesting a spawning from intermediate to total saline environments. Both species have high culturing potentials, considering that their feeding is based on plant and macroinvertebrates, their meat quality and the size they are able to reach. These species represent an interesting resource to the fisher groups of the region and should be integrally assessed. Rev. Biol. Trop. 59 (4): 1717-1728. Epub 2011 December 01.
\end{abstract}

Key words: mugilids, Joturus pichardi, Agonostomus monticola, reproductive biology, Sierra Nevada de Santa Marta, Colombia.

La Sierra Nevada de Santa Marta, declarada por la UNESCO Reserva del Hombre y la Biosfera en 1986, es la formación montañosa litoral más alta del mundo (FPSNSM 1997). En ella, existe una amplia variedad de especies ícticas, muchas de las cuales hacen parte de la pesca de subsistencia de sus habitantes que en su mayoría son campesinos e indígenas. De estas especies, Joturus pichardi y Agonostomus monticola (conocidos por las comunidades locales como "besote" y "rayado", respectivamente), presentan una muy buena preferencia de pesca, debido a las buenas características organolépticas de su carne (Eslava 2009) y las 
tallas que alcanzan en el medio; considerados peces muy populares entre los pescadores fluviales (Garzón \& Wedler 1997).

$J$. pichardi y A. monticola son peces dulceacuícolas que pertenecen a la Familia Mugilidae, la cual incluye generalmente especies marinas y estuarinas (Harrison 2002). Estas dos especies habitan las partes cálidas de los ríos montañosos, donde se presentan corrientes fuertes, grandes rocas y aguas claras y al parecer requieren del agua marina en los estados iniciales de su ciclo de vida (Cruz 1987, Garzón \& Wedler 1997).

Son abundantes los estudios sobre la biología, reproducción y alimentación de los mugílidos, la mayoría ha sido desarrollados en especies marinas y estuarinas, especialmente del género Mugil (e.g. Álvarez-Lajonchere 1976). Para las especies $J$. pichardi y $A$. monticola, consideradas principalmente dulceacuícolas, gran parte de la información relevante, especialmente de su biología reproductiva, proviene de poblaciones centroamericanas y está relacionada con su distribución, caracteres distintivos y aspectos ecológicos (Bussing 1987, Cruz 1987, Phillip 1993, Aiken 1998, Cotta-Ribeiro \& MolinaUreña 2009) pero es poco el conocimiento relacionado con la biología reproductiva de estos mugílidos en las poblaciones colombianas. Este estudio pretende evaluar la biología reproductiva de estos dos mugílidos a partir de indicadores y estados de madurez gonadal de las especies, con el fin de conocer aspectos de su comportamiento reproductivo.

\section{MATERIALES Y MÉTODOS}

Se realizaron muestreos mensuales entre julio 2005 y diciembre 2006, en los ríos Toribío (1102'16" N - 74²'16" W) y Piedras

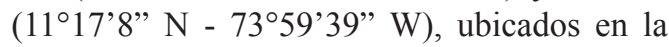
región noroccidental de la SNSM en el departamento del Magdalena y que drenan en el mar Caribe de Colombia. Los individuos fueron capturados en diferentes sitios y horas del día. En los muestreos se utilizaron atarrayas (radios de 3 y $5 \mathrm{~m}$ completamente extendida y una luz de malla de 15 y $20 \mathrm{~mm}$ respectivamente), pituco (arpón artesanal), chinchorros, mantas y redes de mano. Se empleó un equipo de buceo autónomo cuando las condiciones de visibilidad lo permitieron. La utilización de cada arte estuvo determinada por las características ambientales y la facilidad puntual para la captura de los ejemplares. Para el registro de la ubicación geográfica y la altura en metros sobre el nivel del mar (msnm) se utilizó un GPS. Los datos de precipitación mensual y caudal fueron adquiridos a través del Instituto de Hidrología, Meteorología y Estudios Ambientales (IDEAM) de Colombia (IDEAM 2005). En campo, se tomó la temperatura superficial del agua $\left({ }^{\circ} \mathrm{C}\right)$, oxígeno disuelto $(\mathrm{mg} / \mathrm{L})$, alcalinidad $(\mathrm{mg} / \mathrm{L})$, dureza $(\mathrm{mg} / \mathrm{L}), \mathrm{pH}$ y conductividad $(\mu \mathrm{S} / \mathrm{cm})$ con equipos electrónicos de medición. En el río Toribio, el caudal fue estimado haciendo mediciones del ancho del río y el uso de un elemento flotador para obtener la velocidad de la corriente.

La identificación taxonómica de las especies se realizó con base en las claves de Harrison (2002). Cada ejemplar fue evaluado morfométricamente así: longitud total (LT), longitud horquilla (LH) y longitud estándar (LS), al milímetro más cercano con un ictiómetro convencional, y el peso total (WT) al gramo más cercano con una balanza electrónica con capacidad de $2000 \mathrm{~g}( \pm 1 \mathrm{~g})$. Para el análisis de los ejemplares capturados, los peces fueron eviscerados y las gónadas se separaron del resto de órganos, registrándose fecha, sitio de captura, sexo, peso eviscerado, peso de las gónadas y estado de madurez sexual de acuerdo con la escala de Vazzoler (1996). Para el caso del peso de las gónadas se utilizó una balanza analítica con precisión de $\pm 0.001 \mathrm{~g}$.

Estimación de la proporción sexual: $\mathrm{La}$ proporción sexual total y mensual se estimó de acuerdo con la ecuación:

$$
\% \text { hembras }=100 *(\mathrm{Nm} / \mathrm{Nt}) \text {, }
$$

donde $\mathrm{Nm}$ es el número hembras y $\mathrm{Nt}$ es el número total de individuos.

La proporción sexual fue expresada como el número de hembras por cada macho. La 
proporción sexual a la talla se calculó con la técnica de Holden \& Raitt (1975) utilizando intervalos de clase de $20 \mathrm{~mm}$.

Índice gonadosomático (IGS): Se estimó con base en las ecuaciones de Vazzoler (1996) y Tresierra \& Culquichicón (1993), y fue graficado en función del mes de muestreo:

$$
\mathrm{IGS} 1=100 *(\mathrm{WG} / \mathrm{WT}) \text {, }
$$

donde WG es el peso de las gónadas y WT es el peso total del pez.

$$
\text { IGS2 }=100 *(\mathrm{WG} / \mathrm{WE}) \text {, }
$$

donde WE es el peso eviscerado del pez. Este índice se conoce como gonadosomático corregido.

Estimación de las tallas de madurez sexual: La talla media de madurez sexual fue estimada separadamente para hembras y machos, a través de la curva que relaciona la frecuencia relativa de individuos adultos por clase de longitud total, según Sparre \& Venema (1995) y ajustada a la ecuación planteada en Andrade (1998):

$$
P i=\frac{1}{1+e^{-(a+b L T)}},
$$

donde Pi es la frecuencia relativa de individuos maduros, $e$ la base del logaritmo neperiano, a y b los coeficientes estimados por el método de mínimos cuadrados y LT la longitud total.

Diámetro de ovocitos maduros: Por medio de un microscopio de luz con reglilla micrométrica se midió el diámetro de los ovocitos de muestras de gónadas maduras. Los valores fueron expresados como la media \pm desviación estándar.

Estimación de la fecundidad: Luego de pesadas las gónadas, se tomaron submuestras de cada una de ellas $(0.12-0.25 \mathrm{~g})$ y se guardaron en frascos rotulados, los cuales contenían solución de Gilson. La fecundidad total (F) se estimó aplicando el método gravimétrico (Vazzoler 1996) a partir de la siguiente ecuación:

$$
\mathrm{F}=\mathrm{nG} / \mathrm{g} \text {, }
$$

donde $\mathrm{n}$ es el número de ovocitos maduros en la muestra, $G$ es el peso de todos los ovocitos y g es el peso de la muestra. También se estimó la fecundidad relativa con respecto a la talla, peso del pez y peso de las gónadas.

Área de desove: Se realizaron muestreos en la parte baja de los ríos Toribio y Piedras y en su desembocadura, con el fin de capturar ejemplares maduros en estas zonas dado su comportamiento catádromo (Anderson 1957, Cruz 1987, 1989, Phillip 1993, Ditty \& Shaw 1996, Aiken 1998).

Efecto de la salinidad sobre la activación de espermatozoides de $J$. pichardi y A. monticola y su relación con el área de desove: Un bioensayo piloto se realizó para observar el efecto de la salinidad sobre la movilidad espermática y su posible relación con el área de desove de las especies de estudio. Para ello, se utilizaron cuatro individuos maduros (con liberación de esperma a una leve presión en el abdomen) de $J$. pichardi (un individuo) y A. monticola (tres individuos). Los especímenes fueron mantenidos vivos en el Centro de Desarrollo Pesquero y Acuícola de Taganga de la Universidad del Magdalena, registrando su talla y peso. Los animales ayunaron $24 \mathrm{~h}$ para evitar contaminación de las muestras de semen. Para los ensayos en laboratorio, se utilizaron soluciones activadoras distribuidas en salinidades de $0,10,20,30$ y 36 (agua de mar) UPS (unidades prácticas de salinidad), que se obtuvieron a partir de una dilución de agua de mar filtrada con agua destilada. Para la extracción de las muestras de semen de los ejemplares analizados se siguió la metodología propuesta en González \& Díaz (2001). Sobre cada salinidad experimental, con un cronómetro digital se registró el tiempo de activación espermática en segundos desde el momento en que el activador y la gota de semen entraron en 
contacto hasta que la mayoría de los espermatozoides cesaron el movimiento o presentaron un leve temblor (Cruz-Casallas 2001, González \& Díaz 2001). Sumado a ello, se registraron la motilidad espermática, estimación del número de espermatozoides por conteo en cámara Neubauer, prueba de viabilidad con tinción EosinaNigrosina, coloración y viscosidad (Rodríguez 1992, Cruz-Casallas 2001) sobre las muestras de semen evaluadas.

Se utilizó estadística descriptiva y las variables fueron expresadas como promedio \pm desviación estándar. Se estimó la relación entre las variables LT vs F, WT vs F y WG vs F, al igual que los coeficientes de correlación para cada una de ellas. Se utilizó un análisis de varianza a una vía (previa comprobación de los supuestos de normalidad de los datos y homogeneidad de varianzas usando la prueba de Levene) para verificar diferencias entre los estados de maduración de los indicadores IGS1 e IGS2 y entre los tiempos de activación espermática y motilidad evaluados a partir del efecto de salinidad sobre las muestras de semen. Cuando existieron diferencias estadísticas significativas en el ANOVA, se efectuó una prueba post-hoc empleando el test de Tukey para establecer entre cuales tratamientos se presentaron los contrastes. Para comprobar si la proporción sexual estimada se ajustaba a la esperada, se aplicó la prueba G (Zar 1996). Para llevar a cabo los diferentes procedimientos estadísticos se utilizaron los software SPSSC versión 11.5 y Statgraphics Plus $@$ ㄷ versión 4.0. En los análisis se empleó un nivel de confianza del $95 \%$.

\section{RESULTADOS}

Durante el periodo de estudio, las variables fisicoquímicas no sufrieron variaciones relevantes. Sin embargo, los altos valores registrados, principalmente en la conductividad y caudal, estuvieron relacionados con los mayores valores de pluviosidad de la zona. Los rangos de los parámetros fisicoquímicos medidos en los sitios muestreados durante el estudio son presentados en el Cuadro 1. J. pichardi y A. monticola prefieren aguas cristalinas, habitan los rápidos o zonas inmediatamente río abajo donde todavía la corriente es torrentosa y la velocidad es de moderada a fuerte.

Áreas de desove: No se logró la captura de ejemplares de $J$. pichardi durante los muestreos realizados entre la desembocadura y las partes baja próxima a esta zona, en los ríos de estudio en el periodo de septiembre, octubre y noviembre de 2005 y 2006. Especímenes de A. monticola fueron capturados en octubre y noviembre de 2006 en el área de desembocadura cercana a la zona de mezcla del río con el ecosistema marino, diez machos y cuatro hembras todos sexualmente maduros. Posterior a la captura en estas zonas del río, se verificó que

CUADRO 1

Características fisicoquímicas de los sitios de muestreo en los ríos Toribio y Piedras, SNSM, Colombia, julio 2005-diciembre 2006

TABLE 1

Physicochemical characteristics of sampling places in Toribio and Piedras rivers, SNSM, Colombia, July 2005-December 2006

\begin{tabular}{lccccccc} 
& \multicolumn{7}{c}{ Parámetro fisicoquímicos } \\
& $\begin{array}{c}\text { Temperatura } \\
\left({ }^{\circ} \mathrm{C}\right)\end{array}$ & $\begin{array}{c}\text { Oxígeno } \\
(\mathrm{mg} / \mathrm{L})\end{array}$ & $\begin{array}{c}\text { Conductividad } \\
(\mu \mathrm{S} / \mathrm{cm})\end{array}$ & $\begin{array}{c}\text { Alcalinidad } \\
(\mathrm{mg} / \mathrm{L})\end{array}$ & Dureza $(\mathrm{mg} / \mathrm{L})$ & $\mathrm{pH}$ & $\mathrm{Caudal}^{\left(\mathrm{m}^{3} / \mathrm{s}\right)}$ \\
Río Toribio & $21-27$ & $>8$ & $20-138$ & $38.4-51.6$ & $26.6-35.4$ & $7.26-7.74$ & $1.7-27.4$ \\
Río Piedras & $22-27$ & $>8$ & $88-139$ & $17.1-63.0$ & $24.0-51.3$ & $\mathrm{sd}$ & $3.9-18.9 *$ \\
\hline
\end{tabular}

$\mathrm{sd}=$ sin dato. $*$ tomado de IDEAM (2005) 
los machos presentaron inmediata liberación de esperma con una leve presión abdominal y las hembras mostraron el enrojecimiento de la papila genital y abultamiento del abdomen.

Los individuos de $J$. pichardi capturados en los muestreos no mostraron diferencias morfológicas externas entre ambos sexos. En tanto que en A. monticola existen diferencias fenotípicas entre machos y hembras. Los machos son más pequeños, presentan una coloración más clara, un tenue color verde en el dorso y no exhiben el reticulado amarillento interradial de las aletas, propio de las hembras, las cuales presentan el cuerpo más alargado. En los machos la boca es más delgada con respecto a las hembras.

Proporción sexual e índice gonadosomático: Durante el periodo de estudio, en total se capturaron 14 individuos de J. pichardi (tamaño de muestra no representativo) y 320 de A. monticola.

Para J. pichardi se registraron tallas y pesos que oscilaron entre 160 y $550 \mathrm{~mm}$ de longitud total (LT) y 35.0 y $1779.0 \mathrm{~g}$ de peso total (WT). Nueve individuos fueron hembras, tres machos y dos indiferenciados. Se calculó una proporción sexual total de tres hembras: un macho. De los ejemplares analizados, las hembras presentaron las tallas más grandes en comparación con los machos. De A. monticola se obtuvieron tallas y pesos comprendidos en un rango de 45 y $312 \mathrm{~mm}$ de longitud total (LT) y 1.0 y $275.0 \mathrm{~g}$ de peso total (WT). Se presentaron 185 hembras, 81 machos y 54 indiferenciados. La proporción sexual total fue de 2.3 hembras: 1 macho (Gcal: 41.76, $\mathrm{p}<0.05$, g.l.=1). Se observó predominancia de hembras a lo largo del estudio, salvo en septiembre cuando el número de machos sobrepasó el de las hembras (Gcal: 4.936, p<0.05, g.l.=1). Durante junio, julio y octubre, la proporción sexual fue similar a la esperada (1:1) (Fig. 1). La proporción sexual a la talla evidenció una predominancia de los machos con tamaños pequeños hasta el intervalo $97-117 \mathrm{~mm}$ de LT y se observó ausencia de machos en tallas mayores de $200 \mathrm{~mm}$ de LT.

De los individuos de $J$. pichardi capturados que mostraron avanzado estado gonadal, se registró como menor talla el valor correspondiente a $484 \mathrm{~mm}$ LT $(389 \mathrm{~mm}$ LS) para hembras y $251 \mathrm{~mm}$ LT (208mm LS) en machos. El mayor registro fue de $550 \mathrm{~mm}$ LT $(436 \mathrm{~mm}$

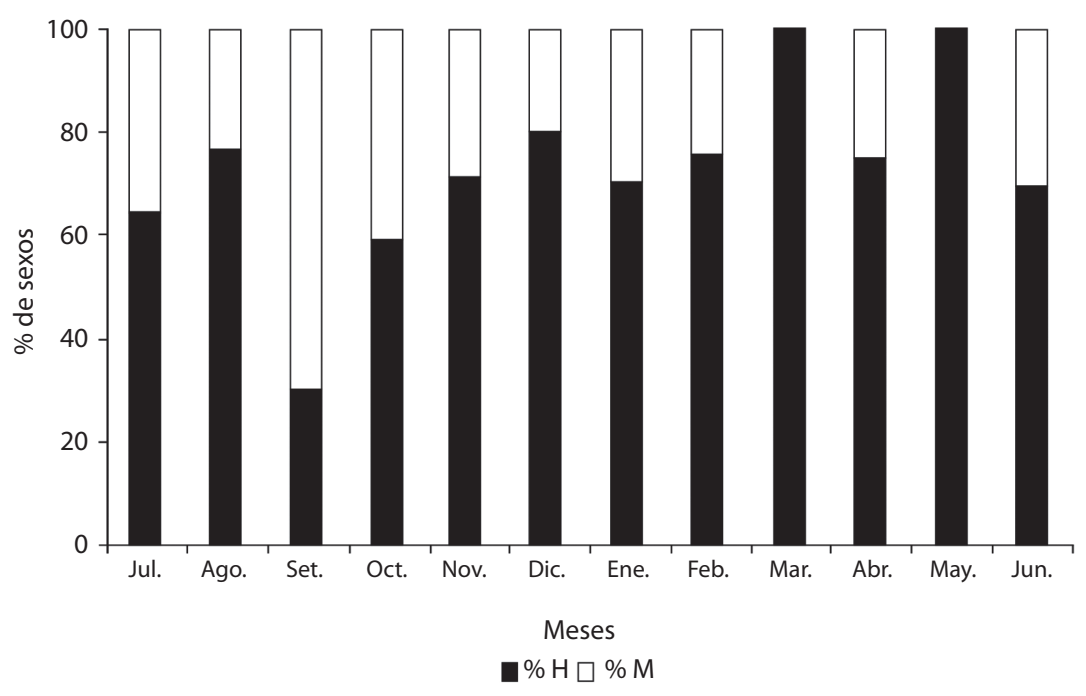

Fig. 1. Proporción sexual mensual de A. monticola en el río Toribio, SNSM, Colombia, julio 2005-junio 2006. Fig. 1. Monthly sex ratio of A. monticola in Toribio river, SNSM, Colombia, July 2005-June 2006. 
LS) y $451 \mathrm{~mm} \mathrm{LT}(356 \mathrm{~mm}$ LS) en hembras y machos, respectivamente. El índice de madurez (IGS) osciló entre 0.16 y 5.19, este último correspondio a un individuo macho maduro de 208mm de LS (Cuadro 2).

\section{CUADRO 2}

Composición de los estadios de madurez gonadal de J. pichardi y A. Monticola, julio 2005-diciembre 2006

\section{TABLE 2}

Composition of gonadal maturity stages of J. pichardi and A. monticola, July 2005-December 2006

\begin{tabular}{lccccc} 
Estado de madurez & I & II & III & IV & Total \\
$J$. pichardi & & & & & \\
$\quad$ Hembras & 2 & 3 & -- & 4 & 9 \\
Machos & -- & -- & 1 & 2 & 3 \\
$\quad$ Indiferenciados & & & & & 2 \\
A. monticola & & & & & \\
$\quad$ Hembras & 94 & 62 & 10 & 19 & 185 \\
$\quad$ Machos & 10 & 38 & 22 & 11 & 81 \\
$\quad$ Indiferenciados & & & & & 54 \\
\hline
\end{tabular}

En cuanto a A. monticola, hembras y machos mostraron un periodo reproductivo entre septiembre y octubre, correspondiente a los mayores valores del IGS, con un periodo final de desove en noviembre. En las hembras el IGS1 y el IGS2 presentaron valores promedio mensuales de $2.33 \pm 1.09$ y $2.77 \pm 1.29$ respectivamente, mientras en los machos fueron de $2.11 \pm 0.79$ y $2.55 \pm 0.96$ para los índices de madurez evaluados. Se encontró diferencia significativa tanto para hembras y machos entre el estado de madurez III (valores más altos del IGS) y los demás estados (estados I, II y IV sin diferencia significativa en IGS1 $(\mathrm{P}=0.07)$ y IGS2 ( $\mathrm{P}=0.182)$ para hembras; estados I, II y IV $(\mathrm{P}=0.346)$ para IGS1 y I-IV $(\mathrm{P}=0.908)$ y II-IV ( $\mathrm{P}=0.134)$ para IGS2 sin diferencia para machos) (Cuadro 3).

\section{Estimación de las tallas de madurez} sexual: Debido a los escasos registros de $J$. pichardi, no se logró inferir la talla de madurez sexual de esta especie. Para $A$. monticola, la talla de inicio de madurez sexual (TIM) encontrada fue de $122 \mathrm{~mm}$ en hembras y $76 \mathrm{~mm}$ de LT en machos. La talla media de madurez sexual (TMM) fue estimada en $172 \mathrm{~mm}$ de LT para hembras (Fig. 2A), en la cual las hembras adultas fueron el $49 \%$ del total de la muestra, mientras que para los machos fue $108 \mathrm{~mm}$ LT (Fig. 2B), y la cantidad de machos adultos representó un $51 \%$ de la muestra total.

Fecundidad: No se lograron registros de fecundidad de J. pichardi. De A. monticola se analizaron 10 hembras en estado III, cuyas tallas y pesos oscilaron entre 138 y $305 \mathrm{~mm}$ de LT (media $210 \pm 57 \mathrm{~mm}$ de LT), y 54 y $263 \mathrm{~g}$

\section{CUADRO 3}

Valores medios del IGS $_{1}$ y GS 2 de A. monticola en el río Toribio, julio 2005-junio 2006

TABLE 3

Mean values of IGS1 and IGS2 of A. monticola in Toribio river, July 2005-June 2006

\begin{tabular}{ccccc} 
& Estado de Madurez & $\mathrm{n}$ & IGS $_{1}^{*}$ & IGS $_{2}{ }^{*}$ \\
Hembras & I & 94 & $0.387 \pm 0.201^{\mathrm{a}}$ & $0.446 \pm 0.225^{\mathrm{a}}$ \\
& II & 62 & $0.985 \pm 0.595^{\mathrm{a}}$ & $1.232 \pm 0.944^{\mathrm{a}}$ \\
\multirow{3}{*}{ Machos } & 10 & $13.164 \pm 0.724^{\mathrm{b}}$ & $18.224 \pm 1.221^{\mathrm{b}}$ \\
& III & 19 & $0.929 \pm 0.268^{\mathrm{a}}$ & $1.074 \pm 0.319^{\mathrm{a}}$ \\
& IV & $0.269 \pm 0.119^{\mathrm{a}}$ & $0.310 \pm 0.134^{\mathrm{a}}$ \\
& I & 10 & $1.765 \pm 1.031^{\mathrm{a}}$ & $2.104 \pm 1.155^{\mathrm{b}}$ \\
& II & 38 & $8.662 \pm 1.754^{\mathrm{b}}$ & $10.654 \pm 1.074^{\mathrm{c}}$ \\
\hline
\end{tabular}

$\mathrm{n}=$ número de ejemplares por estado de madurez. Letras iguales en la misma columna son estadísticamente similares $(\mathrm{a}=0.05)$. * Promedio \pm Desviación estándar. 

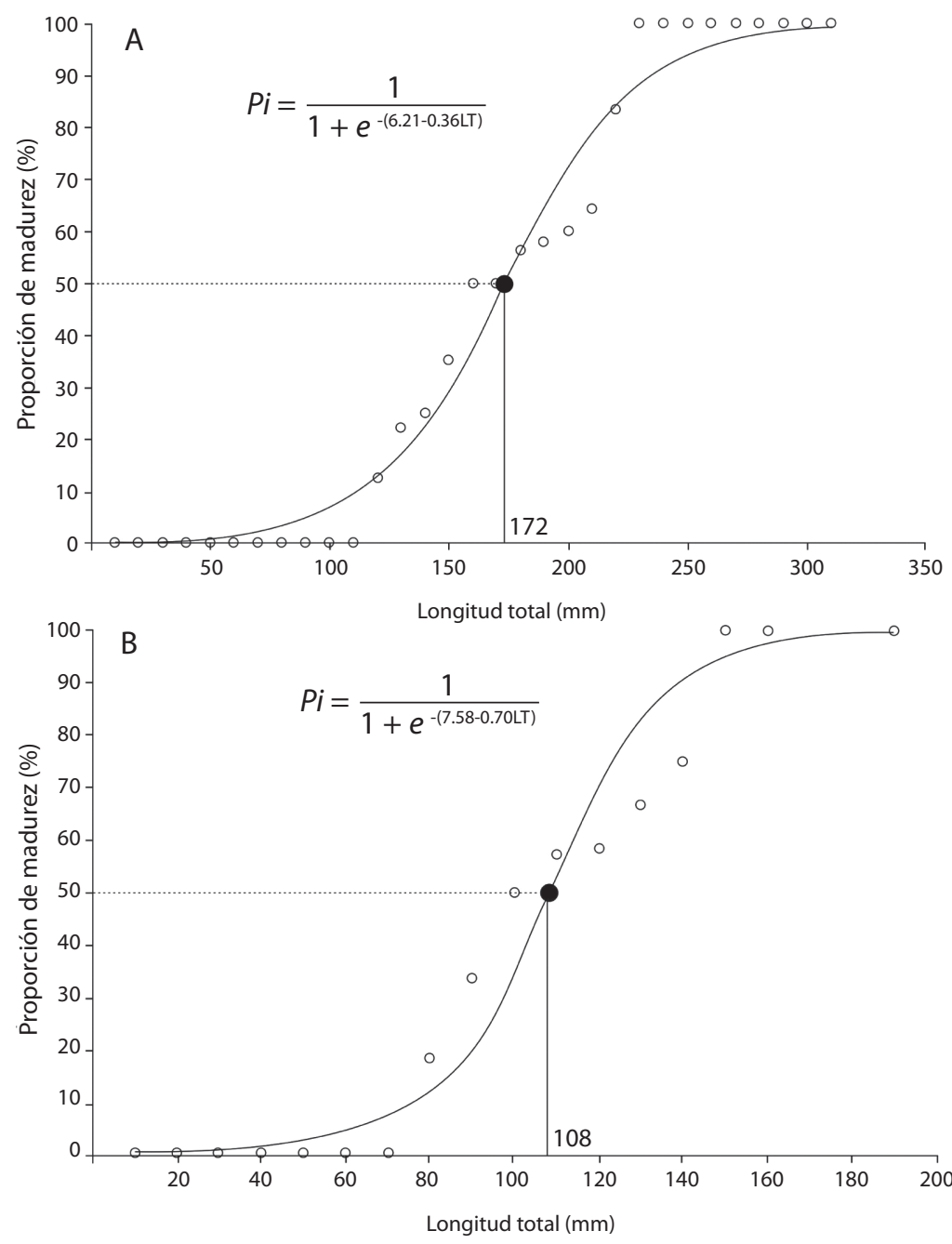

Fig. 2. A. Talla media de madurez sexual para hembras de A. monticola en el río Toribio, SNSM, Colombia. B. Talla media de madurez sexual para machos de A. monticola en el río Toribio, SNSM, Colombia.

Fig. 2. A. Mean size in sexual maturity for females of $A$. monticola in Toribio river, SNSM, Colombia. B. Mean size in sexual maturity for males of A. monticola in Toribio river, SNSM, Colombia.

(media $127 \pm 67 \mathrm{~g}$ ) respectivamente. El peso de las gónadas osciló entre 7.6 y $30.2 \mathrm{~g}$ (media $16.2 \pm 6.4 \mathrm{~g}$ ). La fecundidad total fue estimada en $382075 \pm 153084$ ovocitos, con un mínimo de 197838 para una hembra de $138 \mathrm{~mm}$ LT y peso de 54g, y un máximo de 690112 ovocitos para una hembra de $305 \mathrm{~mm}$ LT y peso de $263 \mathrm{~g}$. Las fecundidades relativas fueron $1790 \pm 385$ ovocitos/mm de LT, $3258 \pm 959$ ovocitos/g y de peso $23925 \pm 4581$ ovocitos/g de gónada. La ecuación que relaciona la fecundidad con el peso fue $\mathrm{F}=1960 \mathrm{WT}-132779(\mathrm{p}<0.05)$ (Fig. 3A), mientras, su relación con respecto a la talla estuvo dada por $\mathrm{F}=395.1 \mathrm{LT}^{1.281}$ (p>0.05) (Fig. 3B).

Diámetro de los ovocitos: En A. monticola se midieron 525 ovocitos, provenientes de 

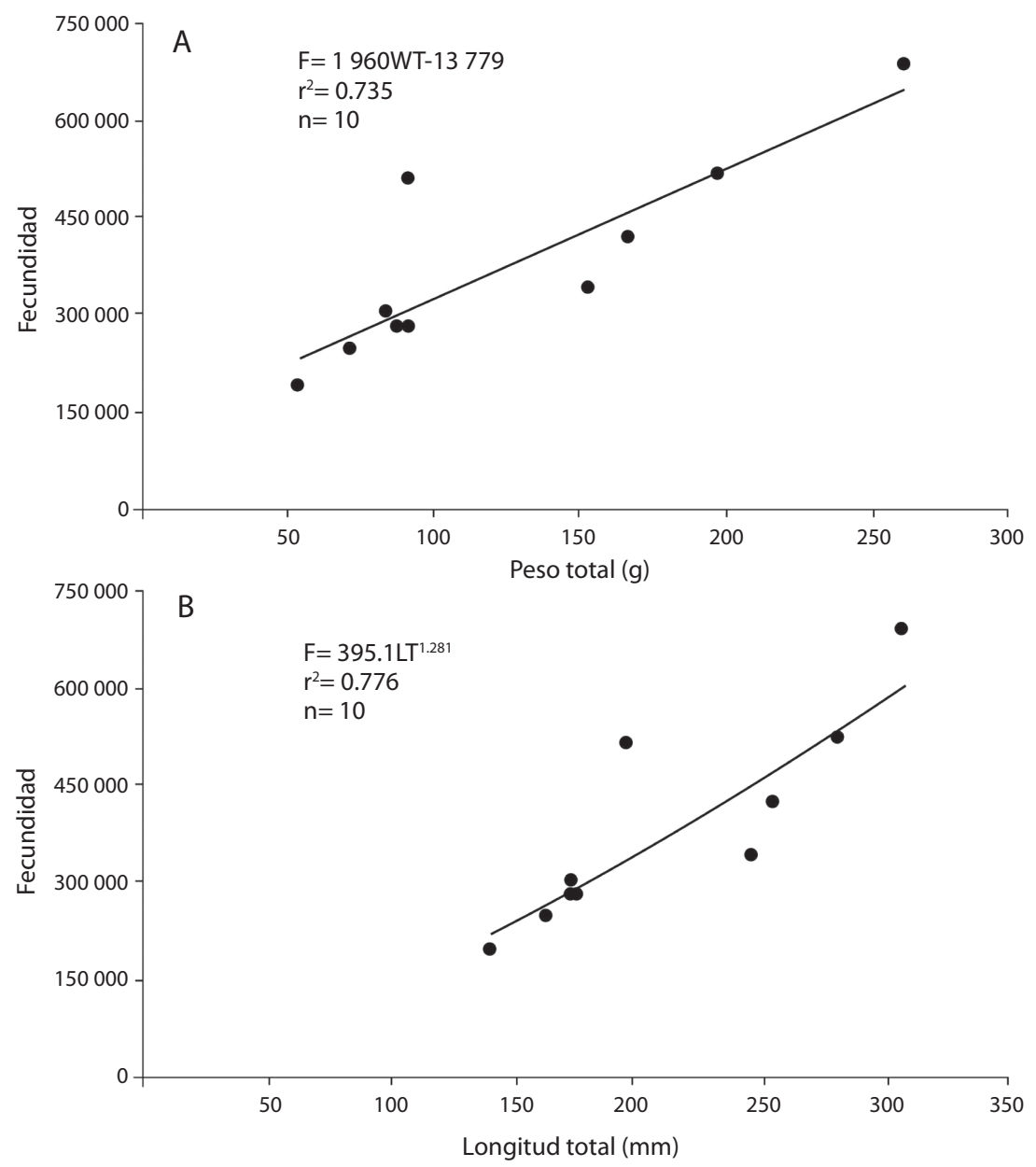

Fig. 3. A. Relación peso total vs. fecundidad en A. monticola del río Toribio, SNSM, Colombia. B. Relación longitud total vs. fecundidad del rayado (A. monticola) del río Toribio, SNSM, Colombia.

Fig. 3. A. Relationship between total weight vs. fecundity for A. monticola in Toribio river, SNSM, Colombia. B. Relationship between total length vs. fecundity for A. monticola in Toribio river, SNSM, Colombia.

gónadas maduras, con un diámetro que osciló entre 270 y $450 \mu \mathrm{m}$, con un valor promedio de $362 \pm 40 \mu \mathrm{m}$.

\section{Efecto de la salinidad sobre la activación} de espermatozoides y su relación con el área de desove: Los resultados sobre el individuo analizado de $J$. pichardi, mostraron que las variables tiempo de activación y motilidad espermática tuvieron los valores más bajos en los tratamientos 0,10 y 30 UPS e inactividad con agua de mar (36 UPS). Sin embargo, el tratamiento con salinidad de 20 UPS se registró un mayor tiempo de activación (845s) y motilidad (90\%).

No se registró movilidad espermática de $A$. monticola en el tratamiento de salinidad 0. En la salinidad de ensayo de 20 UPS se registraron los valores mayores para tiempo de activación y movilidad espermática con $535 \pm 116$ segundos y $97 \pm 3 \%$ respectivamente. El análisis estadístico no reveló diferencias significativas 
entrelas salinidades $10,20,30$ y $36(\mathrm{p}>0.05)$ para las variables evaluadas. Las características de semen para A. monticola fueron: 3 $113 \pm 74 \times 10^{7}$ espermatozoides $/ \mathrm{mL}$, vitalidad de $87.5 \pm 10.5 \%$ (porcentaje de espermatozoides vivos), coloración espermática blanca y viscosidad de $3.4 \pm 0.6$.

\section{DISCUSIÓN}

Evidencias de la distribución en los sistemas fluviales que drenan al mar en Mesoamérica de J. pichardi (Bussing 1987, Cruz 1987, Cruz 1989) y A. monticola (también registrado en Venezuela, Florida e islas del Caribe y Galápagos) han sido ampliamente documentados (Cruz 1987, Phillips 1993, Ditty \& Shaw 1996, Aiken 1998, Cotta-Ribeiro \& MolinaUreña 2009). En Colombia se ha comprobado la presencia $J$. pichardi y A. monticola en los ríos de la vertiente norte de la SNSM (Garzón \& Wedler 1997), evidenciándose en los ríos de estudio, que $J$. pichardi y A. monticola, comparten un hábitat de similares características ecológicas, de corrientes fuertes y claras y zonas pedregosas, que complementan con la zona de desembocadura hacia el mar, en franjas estuarinas, que soportan de forma indirecta su condición migratoria. En relación a esto, Anderson (1957), registra la captura de larvas y pre-juveniles de A. monticola en el mar a un kilómetro de la costa, lo cual fue corroborado posteriormente por Ditty \& Shaw (1996) al recolectar individuos de 3.6 a $25.5 \mathrm{~mm}$ de LS mar afuera en el golfo de México. A la fecha, no existe evidencia que $J$. pichardi entre en el mar, sin embargo, en A. monticola los estudios consideran que la especie migra mar adentro para reproducirse (Ditty \& Shaw 1996, Cruz 1987). En este estudio, esta conducta catádroma estuvo soportada por la captura de prejuveniles recolectados en los meses posteriores al proceso reproductivo, en el área de desembocadura del río e inclusive a pocos metros de mar (lugar conocido como "pluma").

A pesar de no observar dimorfismo sexual en ejemplares de $J$. pichardi, las hembras mostraron un mayor tamaño, coincidiendo con lo encontrado por Cruz (1987). Para A. monticola, Bussing (1987) comenta haber recolectado animales de coloración, tamaño y forma del labio diferente; es posible que en su momento estas características correspondieran a las diferencias entre sexos observadas en este trabajo. Al igual que en $J$. pichardi, la hembras de A. monticola presentaron una longitud mayor. Tal vez, una de las categorías más comunes de dimorfismo sexual en los peces sea la variación del tamaño del cuerpo. Para los mugílidos (e.g.: género $M u g i l$ ), en general las hembras presentan mayor tamaño en relación con sus opuestos masculinos (Vazzoler 1996).

En lo relacionado con la biología reproductiva de las especies, se observó dominancia de hembras y mayores valores de longitud en J. pichardi, lo cual concuerda con Cruz (1987), en estudios sobre poblaciones hondureñas. El autor menciona que la proporción de hembras fue mayor para tallas superiores a $250 \mathrm{~mm}$ de LS. Por otra parte, A. monticola, mostró una mayor proporción de hembras, lo cual es similar a lo reportado en Trinidad por Phillip (1993), quien registra una dominancia de hembras en tallas superiores a $100 \mathrm{~mm}$ de LS y a su vez, difiere de lo encontrado por Aiken (1998), en Jamaica, quien señala una mayor proporción de machos. Vazzoler (1996), considera que en algunas especies de la familia Mugilidae, la relación sexual es varios machos por hembra, lo cual únicamente se evidenció durante los muestreos realizados en el mes de septiembre y en el análisis por tamaño en las tallas pequeñas (individuos menores de $118 \mathrm{~mm} \mathrm{LT}$ ).

Cruz (1987), establece que el periodo de precipitación máxima se encuentra relacionado con el proceso de reproducción en estas especies, lo cual se demostró en los registros de las dos especies estudiadas, observándose que el ciclo de desarrollo gonadal de J. pichardi ha finalizado en diciembre, etapa de post-desove, y que su periodo de desove osciló entre los meses de septiembre a noviembre, periodo con los valores más altos de precipitación para la zona (IDEAM 2005). Las tallas de las hembras de $J$. pichardi, en estado post-desove fueron similares a las anotadas en el trabajo de Cruz 
(1987) en Honduras y éstas presentaron también un mayor tamaño frente a los machos.

Por otro lado, Phillip (1993) en Trinidad, encuentra que el periodo de reproducción para A. monticola ocurre entre junio y octubre, coincidiendo también con la estación lluviosa de la zona. En Jamaica, Aiken (1998), estableció una estación de desove de mayo a julio, periodo que también se relaciona con la época lluviosa. Estas observaciones concuerdan con los resultados de este trabajo, mostrando una sincronía entre el periodo de mayor precipitación (septiembre a noviembre) (IDEAM 2005) y la época de desove de ambas especies.

Aiken (1998), registra una TIM para hembras y machos de A. monticola de 123 y $96 \mathrm{~mm}$ LH, constituyéndose el valor de $72 \mathrm{~mm} \mathrm{LH}$ encontrado en este estudio en los machos, como un referente menor para las poblaciones examinadas. Mientras que en las hembras el resultado fue similar. La TMM de este trabajo se asemeja a lo registrado por Aiken (1998), de $166 \mathrm{~mm} \mathrm{LH}$ en las hembras y diferentes para el caso de los machos (148mm LH). Phillip (1993), solo determina la TMM en las hembras con un valor de $135 \mathrm{~mm}$ de $\mathrm{LH}$, valor diferente a lo hallado en este trabajo.

Las estimaciones de fecundidad para las poblaciones del Caribe colombiano de A. monticola, se aproximaron a lo encontrado por Phillip (1993), quien registra valores de 1000000 ovocitos para $70 \mathrm{~g}$ de gónada, mientras que Aiken (1998) estima un número de 340000 ovocitos para $25 \mathrm{~g}$ de gónada y Cruz (1987) en Honduras determina 300000 ovocitos en $75 \mathrm{~g}$ de gónada. Según Vazzoler \& Rossi-Wongtschowski (1976) y Wootton (1991), la fecundidad puede alterarse por el tipo de desove, duración de la época reproductiva, el grado de madurez de los ovocitos y su reabsorción. Así mismo, consideran que la fecundidad es un parámetro extremadamente influenciado, debido a que sufre cambios por factores fisiológicos, metabólicos y ambientales, siendo un reflejo de la relación entre estos factores sobre cada organismo. De acuerdo con Nikolsky (1963), la fecundidad en especies de peces puede variar de una estación reproductiva a otra por causa de condiciones alimentarias, longevidad y tamaño de la primera madurez sexual. Las consideraciones anteriores pueden verse reflejadas en los resultados de este trabajo, los cuales indican que la longitud total es relativamente homogénea, mientras que el peso total, el eviscerado y de las gónadas no lo son. A. monticola presenta ovocitos de mediano tamaño y alta fecundidad (>10 000 ovocitos/año).

Es posible que la salinidad guarde relación con el lugar de desove y sea condición para llevar a cabo este proceso reproductivo en las especies de estudio. Son variados los factores físicos, químicos y fisiológicos que activan la movilidad espermática y dependen de la especie y del medio donde habitan (Billard 1978, Billard \& Cosson 1986). Al respecto, Cosson et al. (1999), tras realizar una revisión bibliográfica, registran la inducción en medios hipertónicos de la movilidad espermática en seis especies marinas (incluyendo el mugílido Mugilcephalus); así mismo, anotan que en los salmónidos, de comportamiento anádromo, la movilidad espermática puede ser activada en medios hipotónicos, isotónicos y en cierto grado, hipertónicos. En contraste, en especies completamente dulciacuícolas la movilidad espermática es activada en medios hipotónicos (Morisawa \& Suzuki 1980, CruzCasallas et al. 2006, Tabares et al. 2006). De los resultados hallados en este estudio, para $J$. pichardi, es probable que el desove suceda en aguas estuarinas (entre el río y el mar), y que el animal no migre a ambientes completamente marinos. Se tiene conocimiento de la captura de animales maduros a pocos metros de la desembocadura (Cruz 1989), pero en Colombia, aún no existe un registro de su presencia en el mar en alguna fase de su ciclo vital. En $A$. monticola, los resultados sugieren que soporta un amplio rango de salinidad, lo cual coincide con larvas y pre-juveniles recolectados mar adentro (Anderson 1957, Ditty \& Shaw 1996), lo que propone que su freza ocurra en un lugar de intermedia a total salinidad, condición que podría encontrarse en la unión del río con el mar o inclusive en el mar propiamente dicho. 
Sigue siendo escaso el conocimiento acerca de aspectos biológicos y ecológicos sobre las poblaciones colombianas de J. pichardi y A. monticola, compresible en alguna manera para $J$. pichardi, si se tiene en cuenta que su distribución se restringe a los ríos de la vertiente norte de la SNSM y que hasta hace poco se certificó su presencia en Suramérica (Garzón \& Wedler 1997). Estas especies presentan una tendencia migratoria catádroma. Es posible que la freza se lleve a cabo en ambientes estuarinos. Posiblemente exista una disminución en las poblaciones de $J$. pichardi aunque no se conocen las causas, tal vez producto de la presión de pesca y el deterioro de los ambientes. Estas especies presentan un proceso reproductivo condicionado por la época con mayores valores de pluviosidad en la SNSM, tal como se ha reportado en otras latitudes, así como en otros géneros de la familia. Por otro lado, su alimentación a base de vegetales y macroinvertebrados, la calidad de su carne y las tallas que pueden alcanzar hacen de estas especies un potencial de cultivo (Garzón \& Wedler 1997, Eslava 2009), por lo cual, se deben efectuar estudios más profundos que revelen mayor información sobre su comportamiento y reproducción en cautividad.

\section{AGREDECIMIENTOS}

Este estudio fue desarrollado en el marco del proyecto "Estudio sobre la biología, ecología y posibilidades de piscicultura del besote Joturus pichardi (Pisces: Mugilidae) de los ríos de la vertiente norte de la Sierra Nevada de Santa Marta, Colombia" auspiciado por COLCIENCIAS, la Universidad del Magdalena y la Asociación Caoba (Código 335609-13549). Los autores agradecen también a Eberhard Wedler, Francisco Correa Polo y Jhon Perdomo Balaguera.

\section{RESUMEN}

Para los mugílidos de agua dulce Joturus pichardi y Agonostomus monticola se han documentado aspectos ecológicos y de distribución, principalmente para las poblaciones de América Central, sin embargo, hay poca información disponible de sus aspectos reproductivos, especialmente en ambientes de agua dulce de Colombia. Por lo tanto para ambas especies se estudió la biología reproductiva en ríos de la Sierra Nevada de Santa Marta durante julio de 2005 y diciembre de 2006. En total, 14 especímenes de $J$. pichardi y 320 A. monticola fueron capturados. Proporción sexual, índice gonadosomático, talla media de madurez, fecundidad y diámetros de los ovocitos fueron evaluados. Se realizó un bioensayo para observar el efecto de la salinidad sobre la movilidad espermática y su posible relación con el área de desove de las especies. Estos mugílidos comparten un hábitat con características ecológicas similares, de corrientes fuertes y claras y zonas pedregosas. Indicadores de madurez gonadal y evidencias indirectas son presentados y con los cuales se comprueba que el periodo reproductivo se encuentra relacionado con los mayores valores de precipitación de la zona (septiembre a noviembre), así como, la migración catádroma de $J$. pichardi y A. monticola. Estas especies representan un potencial de cultivo, dado su alimentación basada en vegetales y macroinvertebrados, la calidad de su carne y las tallas que pueden alcanzar, por lo cual, se deben efectuar estudios más profundos que revelen mayor información sobre su comportamiento y reproducción en cautividad.

Palabras clave: mugílidos, Joturus pichardi, Agonostomus monticola, biología reproductiva, Sierra Nevada de Santa Marta, Colombia.

\section{REFERENCIAS}

Aiken, A.K. 1998. Reproduction, diet and population structure of the mountain mullet, Agonostomus monticola, in Jamaica, West Indies. Env. Biol. Fish. 53: 347-352.

Álvarez-Lajonchere, L. 1976. Contribución al estudio del ciclo de vida de Mugil curema Valenciennes (Pisces: Mugilidae). Ciencias Serie 8, Invest. Mar. 28: 1-130.

Anderson, W.W. 1957. Larval forms of the fresh-water mullet (Agonostomus monticola) from the open ocean off the Bahamas and south Atlantic coast of the United States. Fish. Bull. 120: 415-425.

Andrade, J.P. 1998. Age and growth of the bastard sole, Microchirusazevia (Capello, 1868) (Pisces, Soleidae) from the south coast of Portugal. Fish. Res. 34: 205-208.

Billard, R. 1978. Changes in structure and fertilizing ability of marine and freshwater fish spermatozoa diluted in media of various salinities. Aquaculture 14: 187-198.

Billard, R. \& M.P. Cosson. 1986. Sperm motility in rainbow trout, Parasalmomykiss; effects of $\mathrm{pH}$ and temperature. Reprod. Fish. 44: 10-12. 
Bussing, W.A. 1987. Peces de las aguas continentales de Costa Rica. Universidad de Costa Rica, San José, Costa Rica.

Cosson, J., R. Billard, C. Cibert, C. Dreanno \& M. Suquet. 1999. Ionic factors regulating the motility of fish sperm, p. 161-186. In C. Gagnon (ed.). The Male Gamete: From basic knowledge to clinical applications. Cache River, Paris, Francia.

Cotta-Ribeiro, T. \& H. Molina-Ureña. 2009. Ontogenic changes in the feeding habits of the fishes Agonostomus monticola (Mugilidae) and Bryconbehreae (Characidae), Térraba River, Costa Rica. Rev. Biol. Trop. 57: 285-290.

Cruz-Casallas, P.E. 2001. Técnicas de laboratorio para la evaluación de la calidad seminal en peces. Rev. Orinoquía 5: 155-163.

Cruz-Casallas, P.E., V.M. Medina-Robles \& Y.M. VelascoSantamaría. 2006. Protocolo para la crioconservación de semen de yamú (Bryconamazonicus Spix\&Agassiz 1829). Rev. Colom. Cienc. Pecua. 19: 146-151.

Cruz, G.A. 1987. Reproductive biology and feeding of Cuyamel Joturus pichardi and Tepemechin Agonostomus monticola (Pisces: Mugilidae) from Rio Platano, Mosquita, Honduras. Bull. Mar. Sci. 40: 63-72.

Cruz, G.A. 1989. Joturus pichardi (Pisces: Mugilidae) sexualmente maduros capturados en Laguna de Brus y en la desembocadura del Río Plátano, Honduras. Rev. Biol. Trop. 37: 107-108.

Ditty, J.G. \& R.F. Shaw. 1996. Spatial and temporal distribution of larval striped mullet (Mugilcephalus) and white mullet (M. curema, Family: Mugilidae) in the Northern gulf of Mexico, with notes on mountain mullet, Agonostomus monticola. Bull. Mar. Sci. 59: 271-288.

Eslava, P.E. 2009. Estimación del rendimiento y valor nutricional del besote Joturus pichardi Poey, 1860 (Pisces: Mugilidae). Rev. MVZ 14: 1576-1586.

FPSNSM-Fundación Pro-Sierra Nevada de Santa Marta. 1997. Plan de desarrollo sostenible de la Sierra Nevada de Santa Marta: Estrategia de conservación de la Sierra Nevada de Santa Marta. Proyecto de cooperación Colombia-Alemán. Colombia, Graficompany.

Garzón, J. \& E. Wedler. 1997. Primer registro del besote Joturus pichardi Poey 1860 (Pisces: Mugilidae) para Colombia y América del Sur. Boletín Ecotrópica 31: 1-9.

González, O.E.S. \& J.A.S. Diaz. 2001. Principios básicos de criopreservación de semen de peces, p. 253-263. In H. Rodríguez, P.V. Daza \& M. Carrillo (eds.).
Fundamentos de Acuicultura Continental. Instituto Nacional de Pesca y Acuicultura (INPA), Colombia.

Harrison, I.J. 2002. Family Mugilidae, p. 1071-1085. In K. Carpenter (ed.). Species identification guide for fishery purposes the living marine resources of the Western Central Atlantic Vol. 2. FAO, Roma.

Holden, M.J. \& D.F.S. Raitt. 1975. Manual de ciencia pesquera. Pesca 2. Métodos para investigar los recursos y su aplicación. Documento Técnico de Pesca No. 115. FAO, Roma.

IDEAM, 2005. Base de datos de precipitación anual total, años 2003 a 2005. Instituto de Hidrología, Metereología y Estudios Ambientales (IDEAM), Bogotá, Colombia.

Morisawa, M. \& K. Suzuki. 1980. Osmolality and potassium ion: their roles in initiation of sperm motility in teleost. Science 210: 1145-1147.

Nikolsky, G. 1963. The ecology of fishes. Academic, Nueva York, EEUU.

Phillip, A.D. 1993. Reproduction and feeding of the mountain mullet, Agonostomus monticola, in Trinidad, West Indies. Env. Biol. Fish. 37: 47-55.

Rodríguez, G.M. 1992. Técnicas de evaluación cuantitativa de la madurez gonádica en peces. México.

Sparre, P. \& S.C. Venema. 1995. Introducción a la evaluación de recursos pesqueros tropicales. Parte 1. Documento Técnico de Pesca No. 306. FAO, Roma.

Tabares, C.J., A.F. Montoya, L. Arboleda, A. Echeverri, L.F. Restrepo \& M. Olivera-Ángel. 2006. Efecto de la pluviosidad y el brillo solar sobre la producción y características del semen en el pez Bryconhenni (Pisces: Characidae). Rev. Biol. Trop. 54: 179-187.

Tresierra, A. \& Z. Culquichicón. 1993. Manual de biología pesquera. Universidad Nacional da Trujillo, Perú.

Vazzoler, A.E.A. de M. 1996. Biología da reproduçâo de peixes teleósteos: teoria e prática. EDUEM, Maringa, São Paulo, Brasil.

Vazzoler, A.E.A. de M. \& C.L.D.B. Rossi-Wongtschowski. 1976. Sardinellabrasiliensis: tipo de desova, fecundidade e potencial reprodutivo relativo. I. Área entre $23^{\circ} 40^{\prime} \mathrm{S}$ e $24^{\circ} 40^{\prime} \mathrm{S}$, Brasil. Bol. Inst. Oceanogr. São Paulo 25: 131-155.

Wootton, R.J. 1991. Ecology of teleost fishes. Chapman \& Hall, Londres, Inglaterra.

Zar, J.H. 1996. Biostatisticalanalysis. Prentice Hall, Nueva Jersey, EEUU. 